\title{
Research Article \\ Performance Comparison of KOMPSAT-5 Precision Orbit Determination with GRACE
}

\author{
Kyoung-Min Roh $\mathbb{D}^{1}$ and Yoola Hwang ${ }^{2}$ \\ ${ }^{1}$ Korea Astronomy and Space Science Institute, Daejeon 34055, Republic of Korea \\ ${ }^{2}$ Electronics and Telecommunications Research Institute, Daejeon 34129, Republic of Korea \\ Correspondence should be addressed to Kyoung-Min Roh; kmroh@kasi.re.kr
}

Received 11 October 2019; Revised 13 January 2020; Accepted 8 February 2020; Published 27 February 2020

Academic Editor: Christian Circi

Copyright (c) 2020 Kyoung-Min Roh and Yoola Hwang. This is an open access article distributed under the Creative Commons Attribution License, which permits unrestricted use, distribution, and reproduction in any medium, provided the original work is properly cited.

\begin{abstract}
The Korean Multipurpose Satellite-5 (KOMPSAT-5) launched on 22 August 2013 was equipped with a global positioning system (GPS) receiver for precision orbit determination (POD). Even though the GPS receiver of KOMPSAT-5 shares the same heritage as the BlackJack receiver onboard in Gravity Recovery and Climate Experiment (GRACE) satellites, KOMPSAT-5 has a lower orbital position accuracy $(\sim 10 \mathrm{~cm})$ compared with GRACE $(\sim 2 \mathrm{~cm})$. The reduced dynamic and kinematic methods are applied for POD of KOMPSAT-5 and GRACE to investigate the GPS observation quality due to the satellite operation concept and hardware design. The results are analyzed in terms of the number of observations and their spatial distribution, GPS signal quality, and orbital position accuracies. The results show that the frequent attitude maneuvers of KOMPSAT- 5 affect the quality of the GPS signals and solutions obtained from the kinematic method compared with that determined from the reduced-dynamic method. The onboard patch GPS antenna installed in KOMPSAT-5 and its geometrical position resulted in more erratic measurement residuals by $140 \%$ compared with the choke ring antenna of GRACE. The POD accuracy is dependent on the hardware design and regular attitude tilting for the synthetic aperture radar (SAR) imaging even though the same GPS receiver performances.
\end{abstract}

\section{Introduction}

Precision orbit determination (POD) plays a critical role for satellite missions such as geodesy, precise image processing, and ground track maintenance. The specification of orbital position accuracy has been tightened to a few centimeters for geodetic missions such as the Gravity Recovery and Climate Experiment (GRACE) and Gravity Field and SteadyState Ocean Circulation Explorer (GOCE) satellites [1, 2]. Onboard Global Positioning System (GPS) receiver is a key element that enables Low-Earth Orbiter (LEO) satellite to have such a high position accuracy. GPS receivers work as stand-alone systems without any ground interferences and give continuous observations that can provide real-time position information. If accumulated observations are processed with a high-fidelity software, more precise orbital positions can be achieved than any other measurement systems. These features make them highly cost-effective and attractive remote sensing systems, and now POD based on GPS obser- vations is common for most LEO missions even in cases where the required levels of orbital position accuracies are not stringent.

The Korean Multipurpose Satellite- (KOMPSAT-) 5 is a satellite equipped with a dual-frequency GPS receiver for POD, which is used for the secondary mission of the satellite. KOMPSAT-5 was launched on 22 August 2013 to an orbit with an altitude of $550 \mathrm{~km}$ and an inclination of $97.6 \mathrm{deg}$. KOMPSAT- 5 consists of two main payloads: (1) Corea SAR Instrument (COSI) and (2) Atmosphere Occultation and Precision Orbit Determination (AOPOD) [3]. COSI is used for the primary mission, which provides high-resolution synthetic aperture radar (SAR) images, and obtains geographical information and monitors natural disasters. The secondary payload (AOPOD) consists of a spaceborne dual-frequency GPS receiver and Laser Retro Reflector Array (LRRA) for POD and GPS radio occultation (RO) measurements [4]. The GPS receiver of KOMPSAT- 5 is the Integrated GPS and Occultation Receiver (IGOR), which is the commercial 


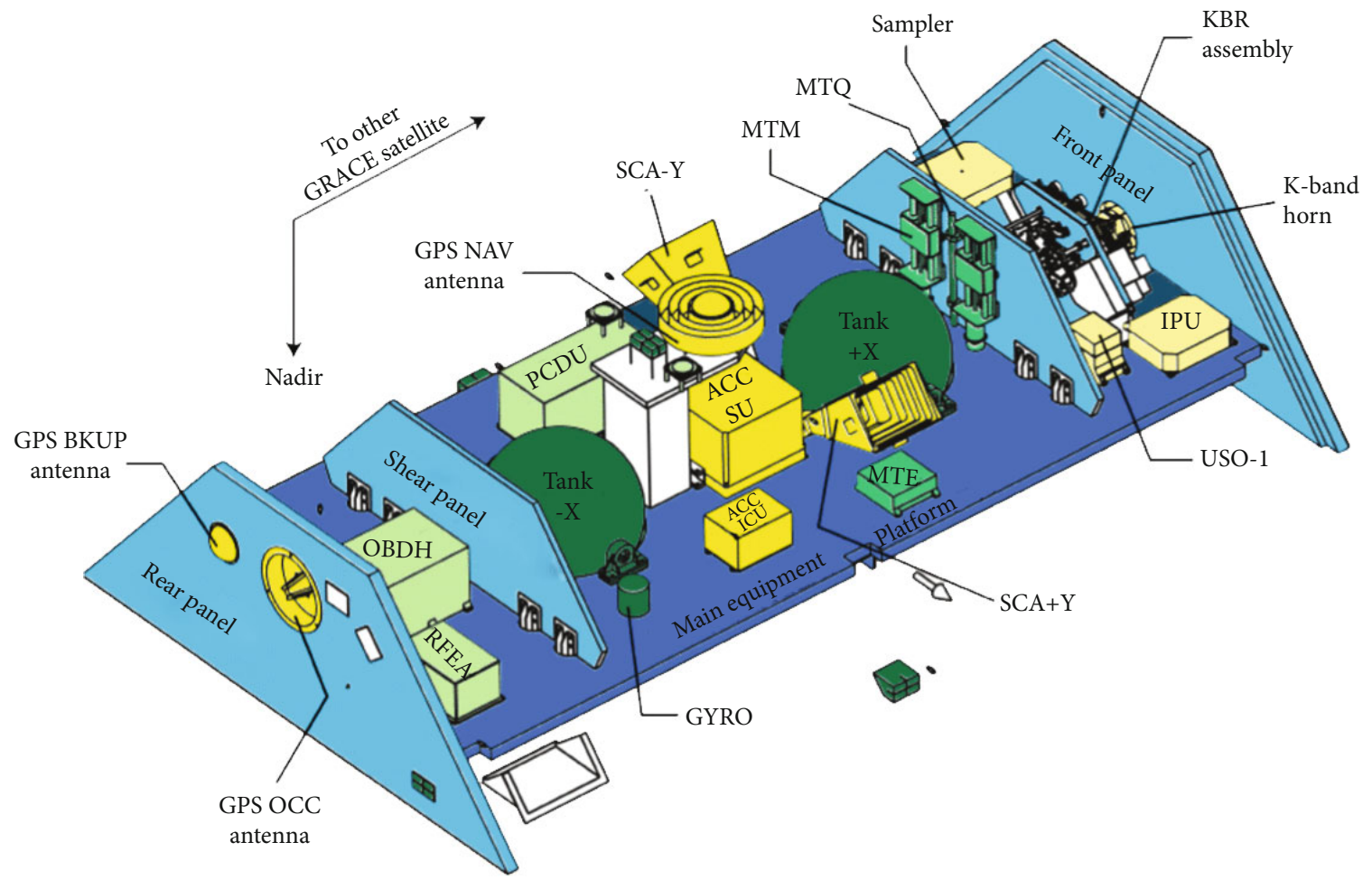

FIGURE 1: Position of GPS antenna position and type of the GRACE spacecraft [7].

version of the BlackJack receiver equipped in Challenging Minisatellite Payload (CHAMP), GRACE, and TerraSAR-X [5]. However, since the POD receiver of KOMPSAT-5 focuses on these two major tasks such as SAR imaging and RO, whose required orbital accuracy is about the level of $20 \mathrm{~cm}$ [6], the operational strategy and satellite design are not optimized to attain the cm-level POD accuracy. For example, GRACE, which is dedicated for geodetic missions, is equipped with a choke ring GPS antenna to minimize multipath errors [5]. The physical shapes of the antenna are also different, as shown in Figure 1 [7]. GRACE, which is inherited from CHAMP, has a simple box shape without wings, whereas KOMPSAT-5 has a radar array for SAR imaging and solar panels. KOMPSAT-5 performs attitude maneuvers when the satellite passes over the Korean Peninsula for SAR imaging, and the detailed information for these attitude maneuvers is not available to the public, whereas the information for the GRACE satellites such as the antenna reference points, mass for fuel consumption, and attitude solutions from the star sensors are released publicly and they are used in improving the POD results. Based on these differences, it cannot be simply assumed that the orbital position accuracy of KOMPSAT- 5 would be the same as that for GRACE even though the GPS receivers of both satellites share the same heritage. Therefore, it is worth to investigate and compare the POD performance of KOMPSAT-5 with results from geodetic missions like GRACE.

The main objective of this study is to investigate the performance of the GPS-based POD of KOMPSAT-5 and possible factors that affect the POD performance. To this purpose, the POD methods and results obtained for KOMPSAT- 5 are analyzed and compared with those for GRACE. There are several reasons that GRACE was chosen as a reference
POD in this study. First, the orbital characteristics of GRACE, i.e., $500 \mathrm{~km}$ of altitude and $89.0 \mathrm{deg}$ of inclination, are similar to those of KOMPSAT-5 $(550 \mathrm{~km}$ of altitude and $97.6 \mathrm{deg}$ of inclination). Secondly, the GPS receivers of both satellites share the same heritage [5]. Lastly, the POD performance of GRACE has been studied for many years and it has been proven that GRACE has one of the best POD accuracies $[8,9]$. GRACE satellites were also equipped with an additional geodetic instrument to measure intersatellite range and range rate, i.e., K-Band Ranging (KBR). However, the KBR system has no contribution to the POD of a single satellite. Therefore, the POD based on only GPS observations are considered in this study. By comparing these two systems, the POD based on the onboard GPS system and the possible factors that affect the POD performance can be analyzed.

The remainder of this paper is organized as follows. The characteristics of the GPS measurements for KOMPSAT-5 are presented in Section 2. In Sections 3 and 4, the POD scheme and the results pertaining to the POD performance of KOMPSAT- 5 are analyzed and compared with those of GRACE. The effects on the POD performance of antenna type and attitude maneuvers of KOMPSAT-5 are also presented and discussed. Lastly, the conclusions are presented in Section 5.

\section{Characteristics of KOMPSAT-5 GPS Observation}

The IGOR GPS receiver of KOMPSAT-5 was equipped for two main purposes: (1) determination of the precise orbital position of the satellite and (2) measurements of GPS radio 


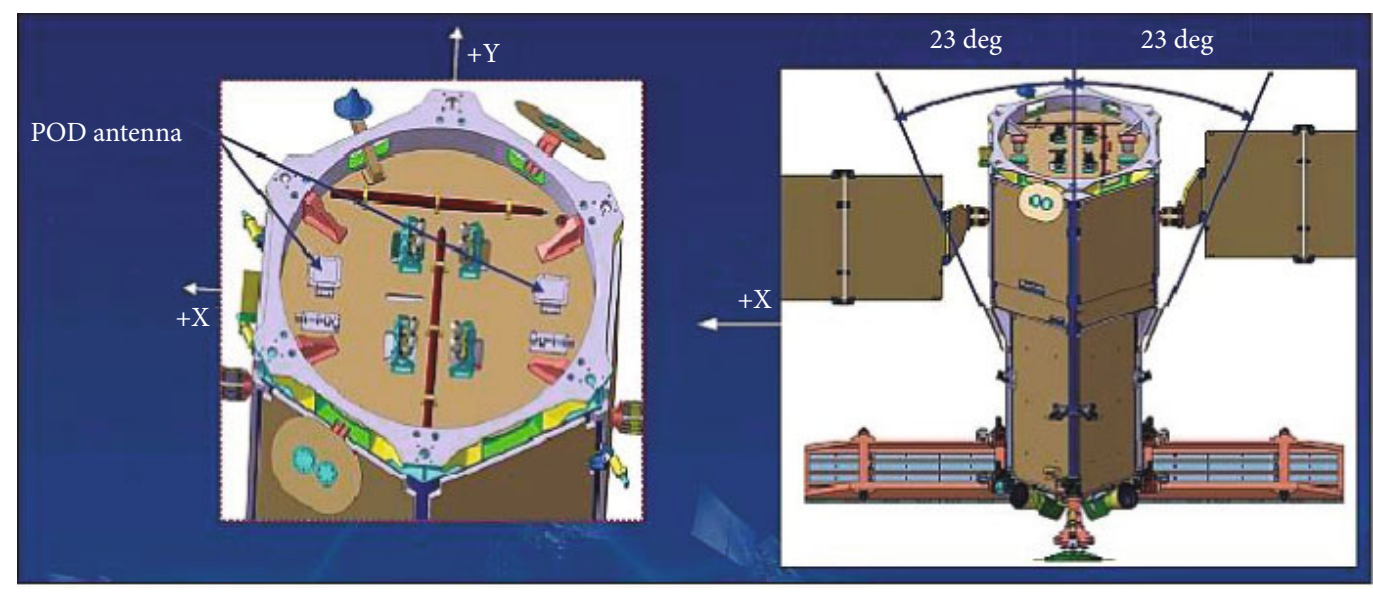

FIGURE 2: GPS antenna configuration for KOMPSAT-5 [10].

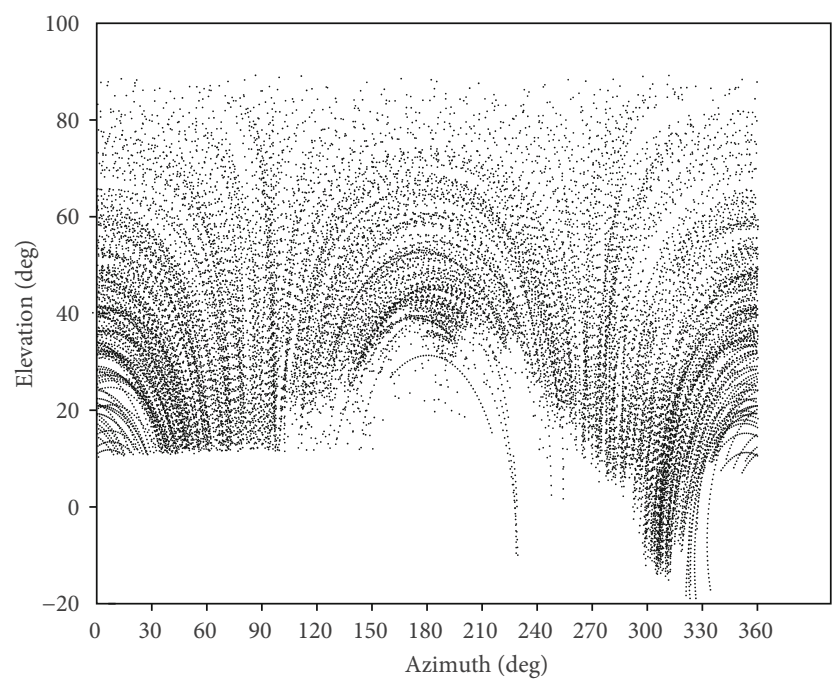

(a)

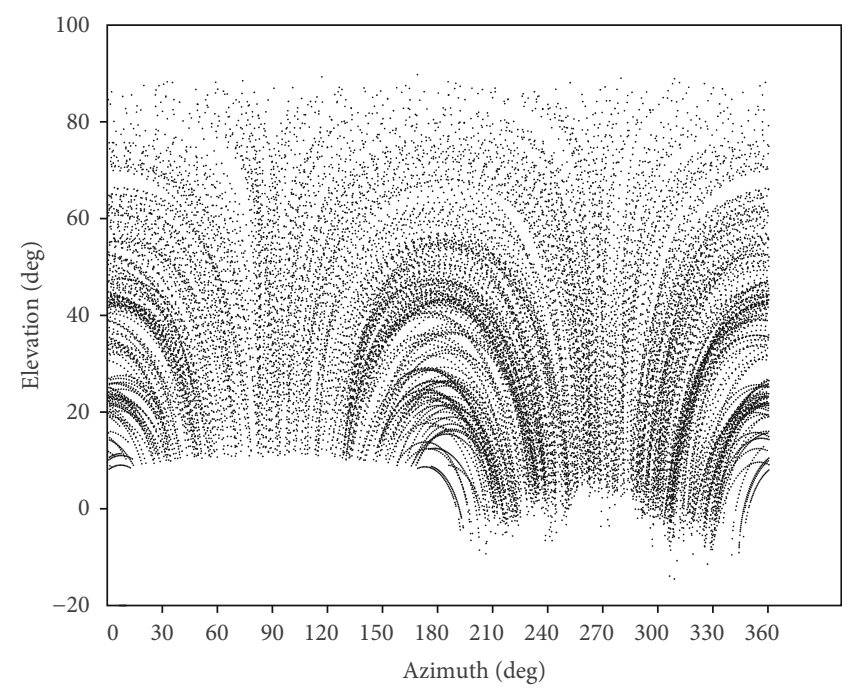

(b)

FIgUre 3: Azimuth-elevation maps of GPS signals for (a) KOMPSAT-5 and (b) GRACE-A.

occultation signals for atmospheric studies [4]. The GPS receiver consists of 48 channels from four antenna inputs, where two inputs are used for POD whereas the other two inputs are used for radio occultation [3]. Two radio occultation antennas are located at the side of the spacecraft to capture signals from the horizontal direction while the antennas for POD are located on the top side of the spacecraft (i.e., zenith directions), as shown in Figure 2. Two types of measurements were made with different sampling rates: (1) $0.1 \mathrm{~Hz}$ (POD) and (2) $50 \mathrm{~Hz}$ (radio occultation). The primary difference between the KOMPSAT- 5 and GRACE spacecraft is related with antenna type and geometrical location. First, the latter are equipped with a patch GPS antenna without a choke ring whereas the former is equipped with a choke ring. The choke ring-type antenna is particularly advantageous to mitigate multipath errors. Another important difference is the geometrical location of the antennas. The choke ring antenna used in the GRACE spacecraft is positioned in such a way to minimize signal blocking by the spacecraft itself (Figure 1). In the case of KOMPSAT-5, two POD antennas are located at the top layer but are installed in locations where there is a lot of interference with other instruments as can be seen in Figure 2. In addition, the receiver is not able to recognize which antenna received a GPS signal. Therefore, the phase offset for each GPS signal cannot be considered during the POD process and it works as measurement noise.

The differences between KOMPSAT-5 and GRACE due to the position and type of GPS antenna are apparent from the azimuth-elevation maps of the GPS signals on 2 January 2016 in Figure 3. The azimuth-elevation maps shown in Figure 3 are obtained based on local-horizon and local-vertical frame, i.e., a nadir-pointing spacecraft without attitude maneuvers. There is a large hole in the GPS signal distribution for KOMPSAT-5, and therefore, the GPS antenna is unable to acquire signals near azimuths of 150 $240^{\circ}$ and elevations of $10-40^{\circ}$. This hole is caused by geometrical issue such as physical blocking by other parts of the spacecraft body. In addition, there is a large number of signals from below the horizon, which is likely contributed by multipath and attitude tilting to take SAR image. As 
mentioned previously, KOMPSAT-5 performs attitude maneuvers regularly $2 \sim 3$ times a day when the satellite passes over the Korean Peninsula in order to capture SAR images, which is the primary investigation of the satellite. These frequent attitude maneuvers affect the quality of the GPS signals in various ways. Specifically, the GPS antenna captures the signals from near the horizon and these signals contain more errors compared with those from the zenith direction due to the long signal propagation distance and atmospheric effects. The characteristics of the GPS observations (specifically, the number of observations per epoch, the percentage of the rejected observations due to errors, and the resulting measurement residuals) were investigated in the later section.

\section{POD Scheme}

The methods and processes used for the POD of KOMPSAT-5, namely, the reduced-dynamic (RD) and kinematic (KIN) methods, are presented in this section. The same POD scheme using only GPS data is applied to the GRACE satellite for the orbit accuracy comparison and verification purposes. The POD solution is achieved using Bernese GNSS software version 5.2 [11], which is based on the reduced-dynamic method, which uses the reduced form of orbital equations of motions. Therefore, the reduceddynamic method has to incorporate an additional parameter estimation to cover the unmodeled effects. In general, the reduced-dynamic method includes the piece-wise constant accelerations (also known as pseudostochastic pulses) to state variables to overcome insufficiencies of the perturbation models like solar radiation pressure and atmospheric drag (in this research, we use traditional POD schemes without considering onboard accelerometer). These accelerations are set up to three directions of radial, along-track, and cross-track. The degree of stochastic pulse density was also investigated to optimize the POD method by various studies $[12,13]$. Spacecraft position by the kinematic method is calculated by least square using only GPS observations epoch by epoch; therefore, it does not need any dynamics of satellite motion; thus, the results are definitely susceptible to the quality of GPS observations.

The POD process developed in this study consists of four steps: (1) determine the preliminary orbit, (2) screen and edit the outlier observations, (3) estimate the final orbit using the reduced-dynamic approach, (4) calculate the kinematic orbit using the same observation data. The POD process was applied to the GRACE-A satellite, and the results were verified by comparing with the officially released orbits by the GRACE science team as a truth [14]. The orbital positions obtained from the reduced-dynamic and kinematic methods were also compared to verify the POD accuracy and assess the quality of GPS data.

Since the objective of this study was to investigate the POD performance of KOMPSAT-5, the same POD scheme was used to determine the orbital positions of the KOMPSAT-5 and GRACE satellites. Namely, the same settings were used for the observation type, sampling interval, frequencies of the pseudostochastic pulses, and direction of both satellites.
TABLE 1: POD schemes for both KOMPSAT-5 and GRACE satellites.

\begin{tabular}{|c|c|}
\hline Observation type & L3 (ionosphere-free combination) \\
\hline Data arc & 24 hours \\
\hline Sampling interval & 30 seconds \\
\hline $\begin{array}{l}\text { Attitude file, phase } \\
\text { center offset, } \\
\text { and variation }\end{array}$ & Not applied \\
\hline Elevation cutoff angle & $5^{\circ}$ \\
\hline $\begin{array}{l}\text { Earth orientation } \\
\text { parameters and } \\
\text { the orbits of the GPS } \\
\text { satellites }\end{array}$ & $\begin{array}{l}\text { Final products from Center for Orbit } \\
\text { Determination in Europe (CODE) [15] }\end{array}$ \\
\hline Geopotential model & $\begin{array}{c}\text { Gravity (EGM2008) } \\
\text { Ocean tide (FES2004) [16] }\end{array}$ \\
\hline Planetary ephemeris & Planetary ephemeris (DE405) [16] \\
\hline Estimation parameters & $\begin{array}{l}\text { Six osculating elements, three } \\
\text { constant empirical accelerations, and } \\
\text { a pseudostochastic pulse-piece-wise } \\
\text { constant acceleration in the radial, } \\
\text { along-track, and cross-track } \\
\text { directions-for every } 6 \text { min. } \\
\text { (empirical acceleration and } \\
\text { pseudostochastic pulse are only applied } \\
\text { to the reduced dynamic method) }\end{array}$ \\
\hline
\end{tabular}

The detailed configurations of the POD method are listed in Table 1.

The POD method was applied to the GPS observations of GRACE-A which is one of the twin satellites in the GRACE mission. The orbital positions were determined for six days beginning from 12 July 2016, and the dates were arbitrarily chosen because there were no special events. The POD results were verified by (1) comparing the solutions obtained from different POD methods, such as reduced-dynamic and kinematic methods, and (2) comparing the solutions with official products. The POD results for GRACE-A are summarized in Table 2, along with the total number of observations. The root-mean-square (RMS) for each direction in Table 2 is calculated using the following equations:

$$
\mathrm{RMS}_{\mathrm{RD}-\mathrm{KIN}}=\sqrt{\frac{1}{N} \sum_{i}^{N}\left(\mathbf{r}_{\mathrm{RD}, i}-\mathbf{r}_{\mathrm{KIN}, i}\right)^{T} \cdot\left(\mathbf{r}_{\mathrm{RD}, i}-\mathbf{r}_{\mathrm{KIN}, i}\right)}
$$

where $N$ is the total number of epoch and the abbreviations $\mathrm{RD}$ and KIN represent the solutions obtained from the reduced-dynamic (RD) and kinematic (KIN) methods, respectively. The calculated RMS is projected to radial, along-track, and cross-track directions. Here, the orbit generated by the science team is called SCI. The RD-SCI stands for the differences between the reduced dynamics and officially released solutions.

The average RMS of the orbital position differences in three-dimension (3D) for RD-KIN, and RD-SCI for six days are $3-5 \mathrm{~cm}$ and $2-3 \mathrm{~cm}$, respectively. The results agree well 
TABLE 2: Comparison of the precise orbit products for GRACE-A satellite.

\begin{tabular}{|c|c|c|c|c|c|c|c|c|c|c|c|c|}
\hline \multirow{2}{*}{ Date } & \multicolumn{4}{|c|}{$\mathrm{RMS}_{\mathrm{RD}-\mathrm{KIN}}(\mathrm{cm})$} & \multicolumn{4}{|c|}{$\mathrm{RMS}_{\mathrm{RD}-\mathrm{SCI}}(\mathrm{cm})$} & \multirow[t]{2}{*}{ \# input obs. } & \multirow[t]{2}{*}{ \# marked obs. } & \multicolumn{2}{|c|}{$\begin{array}{l}\text { Residuals } \\
\text { (mm) }\end{array}$} \\
\hline & Radial & Along-track & Cross-track & $3 \mathrm{D}$ & Radial & Along-track & Cross-track & $3 \mathrm{D}$ & & & $\mathrm{RD}$ & KIN \\
\hline 2016-07-12 & 2.78 & 2.17 & 1.28 & 3.75 & 0.90 & 1.38 & 0.81 & 1.84 & 24832 & 1833 & 5.7 & 3.5 \\
\hline $2016-07-13$ & 2.69 & 2.28 & 1.52 & 3.84 & 0.86 & 1.43 & 0.84 & 1.87 & 25460 & 1864 & 5.7 & 3.6 \\
\hline 2016-07-14 & 3.47 & 3.52 & 1.47 & 5.16 & 0.90 & 1.54 & 0.99 & 2.04 & 25320 & 1981 & 5.8 & 3.6 \\
\hline 2016-07-15 & 2.67 & 2.28 & 1.51 & 3.82 & 1.00 & 1.57 & 0.95 & 2.09 & 24970 & 1819 & 5.8 & 3.5 \\
\hline $2016-07-16$ & 3.00 & 2.38 & 1.46 & 4.10 & 1.43 & 1.65 & 0.95 & 2.38 & 25079 & 1927 & 5.6 & 3.5 \\
\hline 2016-07-17 & 2.79 & 2.50 & 1.13 & 3.91 & 0.93 & 1.52 & 0.99 & 2.04 & 25199 & 1989 & 5.7 & 3.6 \\
\hline
\end{tabular}

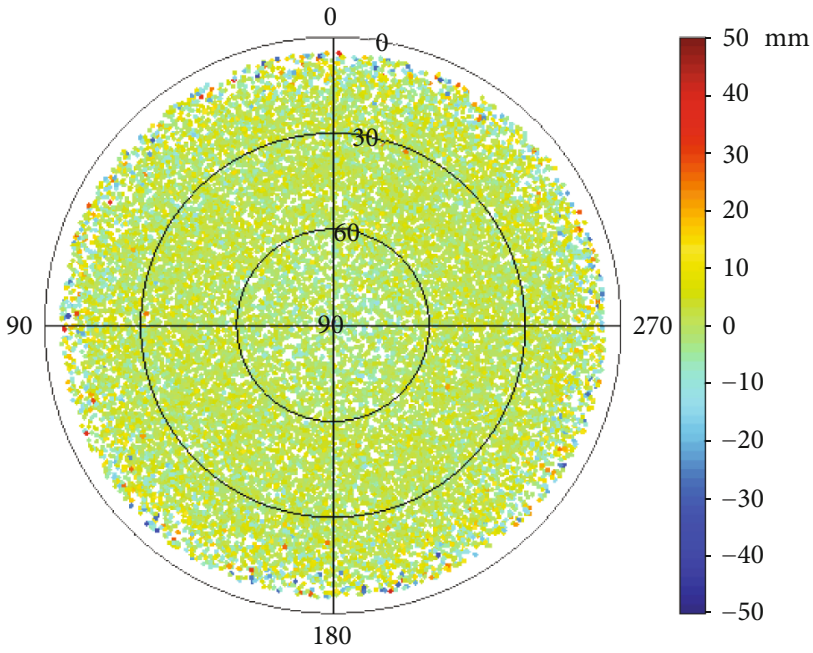

(a)

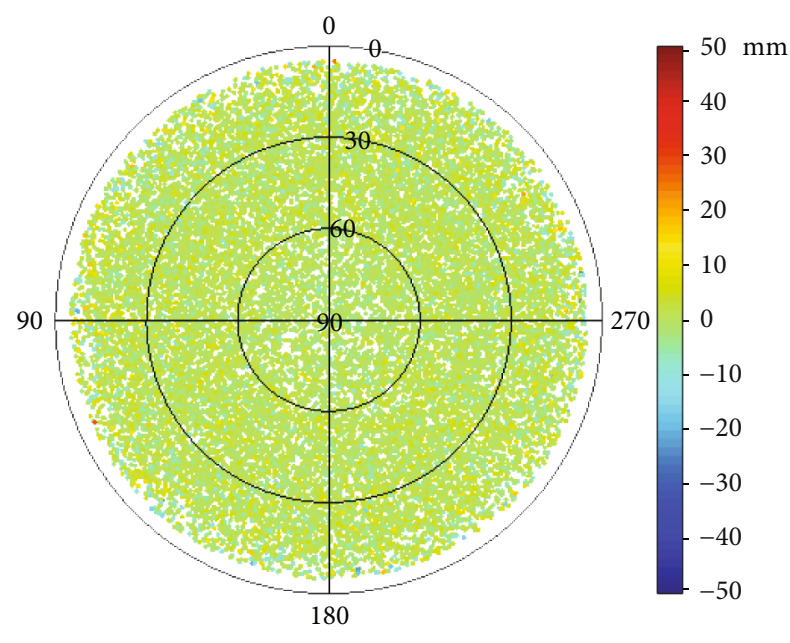

(b)

FIgURE 4: Measurement residuals of GRACE-A: (a) reduced-dynamic and (b) kinematic methods on 12 July 2016.

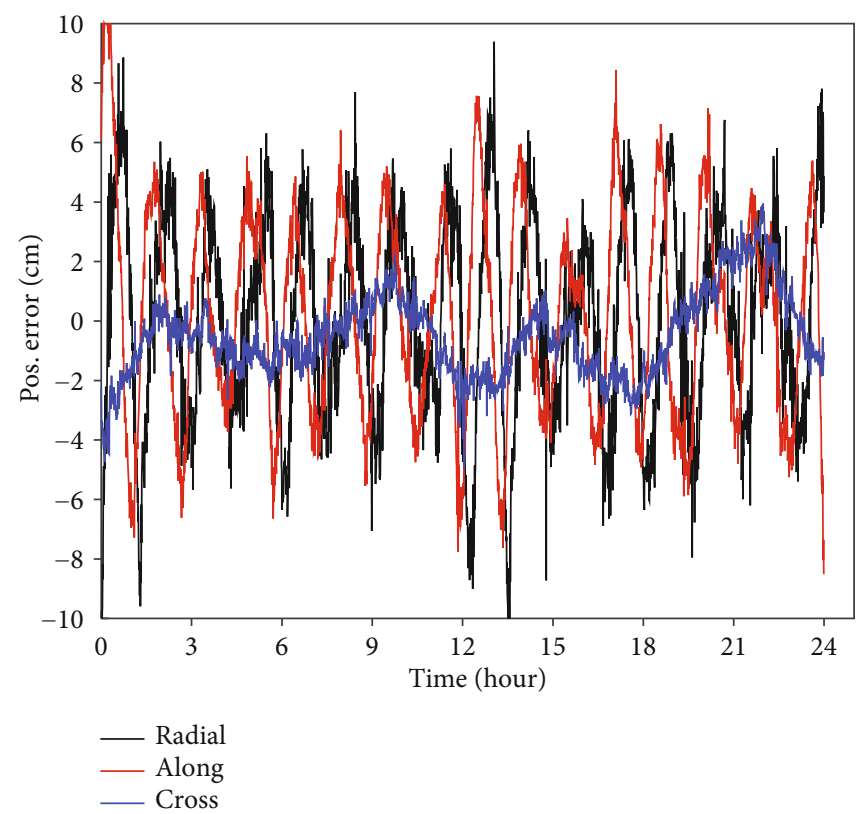

(a) RD-KIN

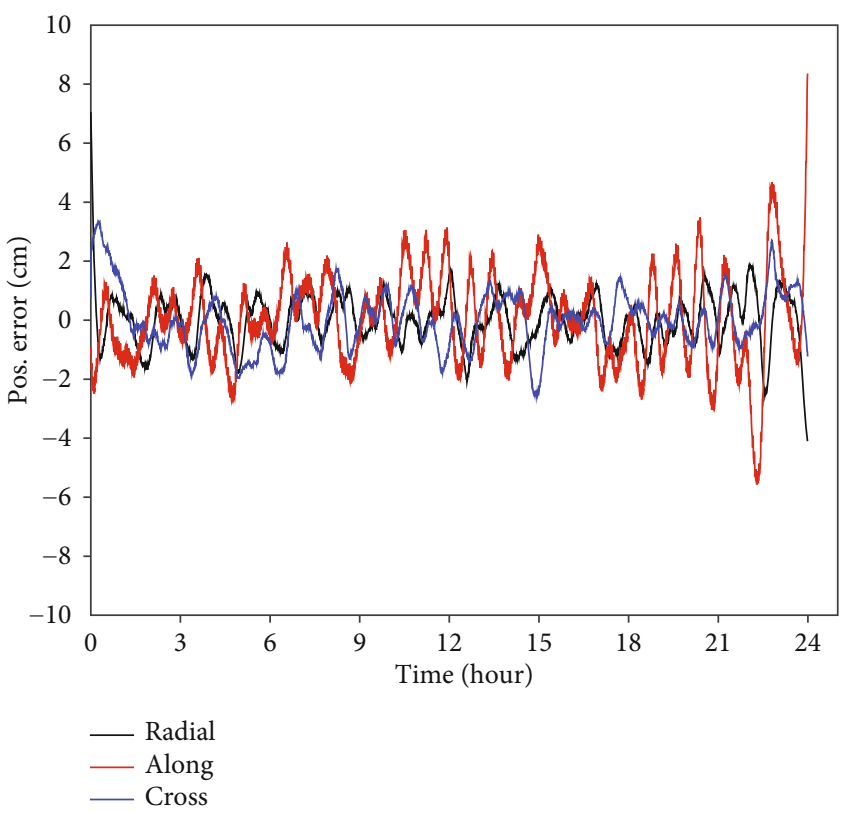

(b) RD-SCI

FIGURE 5: Position errors of the GRACE-A satellite in the radial, along-track, and cross-track directions obtained on 14 July 2016. 
TABLE 3: Start and end time for the actual attitude maneuvers and the removed measurements.

\begin{tabular}{|c|c|c|c|c|c|}
\hline \multirow{2}{*}{ Date } & \multicolumn{2}{|c|}{ Attitude maneuver (hh:mm:ss) } & \multicolumn{3}{|c|}{ Measurement removal (hh: $\mathrm{mm}: \mathrm{ss})$} \\
\hline & Start time & End time & Start time & End time & Duration \\
\hline \multirow{2}{*}{ 2016/07/12 } & $09: 48: 51$ & $10: 31: 25$ & $9: 47: 50$ & $10: 32: 30$ & $00: 44: 40$ \\
\hline & $20: 32: 11$ & $21: 14: 45$ & $20: 31: 10$ & $21: 15: 50$ & $00: 44: 40$ \\
\hline \multirow{3}{*}{ 2016/07/13 } & $09: 45: 19$ & $10: 27: 53$ & $9: 44: 10$ & $10: 29: 00$ & $00: 44: 50$ \\
\hline & $16: 09: 27$ & $16: 52: 01$ & $16: 08: 20$ & $16: 53: 00$ & $00: 44: 40$ \\
\hline & $20: 28: 47$ & $21: 11: 21$ & $20: 27: 50$ & $21: 12: 20$ & $00: 44: 30$ \\
\hline \multirow{2}{*}{ 2016/07/14 } & $09: 42: 01$ & $10: 24: 35$ & $9: 41: 00$ & $10: 25: 40$ & $00: 44: 40$ \\
\hline & $20: 25: 25$ & $21: 07: 59$ & $20: 24: 20$ & $21: 09: 00$ & $00: 44: 40$ \\
\hline \multirow{2}{*}{ 2016/07/15 } & $09: 38: 33$ & $10: 21: 07$ & $9: 37: 30$ & $10: 22: 10$ & $00: 44: 40$ \\
\hline & $20: 22: 02$ & $21: 04: 36$ & $20: 21: 00$ & $21: 04: 40$ & $00: 43: 40$ \\
\hline \multirow{2}{*}{ 2016/07/16 } & $09: 35: 09$ & $10: 17: 43$ & $9: 34: 00$ & $10: 18: 50$ & $00: 44: 50$ \\
\hline & $20: 18: 50$ & $21: 01: 24$ & $20: 17: 50$ & $21: 02: 30$ & $00: 44: 40$ \\
\hline \multirow{2}{*}{ 2016/07/17 } & $09: 31: 51$ & $10: 14: 25$ & $9: 30: 50$ & $10: 15: 30$ & $00: 44: 40$ \\
\hline & $20: 15: 29$ & $20: 58: 03$ & $20: 14: 20$ & $20: 59: 10$ & $00: 44: 50$ \\
\hline
\end{tabular}

TABLE 4: POD results for KOMPSAT-5 with GPS observations during attitude maneuvers.

\begin{tabular}{|c|c|c|c|c|c|c|c|c|c|c|c|c|}
\hline \multirow{3}{*}{ Date } & \multicolumn{12}{|c|}{ Case 1: raw measurement files } \\
\hline & \multicolumn{4}{|c|}{$\mathrm{RMS}_{\mathrm{RD}-\mathrm{KIN}}(\mathrm{cm})$} & \multicolumn{4}{|c|}{$\mathrm{RMS}_{\mathrm{RD}-\mathrm{SCI}}(\mathrm{cm})$} & \multirow{2}{*}{ \# input obs. } & \multirow{2}{*}{ \# marked obs. } & \multicolumn{2}{|c|}{$\begin{array}{l}\text { Residual. } \\
\text { (mm) }\end{array}$} \\
\hline & Radial & Along-track & Cross-track & $3 \mathrm{D}$ & Radial & Along-track & Cross-track & $3 \mathrm{D}$ & & & $\mathrm{RD}$ & KIN \\
\hline 2016-07-12 & 15.57 & 13.67 & 26.91 & 33.96 & 4.34 & 5.15 & 6.39 & 9.28 & 20937 & 3154 & 10.4 & 5.4 \\
\hline $2016-07-13$ & 23.41 & 25.54 & 42.98 & 55.21 & 5.30 & 6.98 & 4.23 & 9.73 & 21010 & 3924 & 10.6 & 5.2 \\
\hline 2016-07-14 & 15.85 & 14.29 & 44.57 & 49.42 & 4.59 & 6.01 & 5.47 & 9.33 & 20956 & 3203 & 10.0 & 5.4 \\
\hline 2016-07-15 & 15.19 & 14.72 & 40.54 & 45.73 & 4.31 & 5.18 & 6.47 & 9.34 & 20959 & 3172 & 10.4 & 5.3 \\
\hline 2016-07-16 & 20.14 & 16.94 & 37.31 & 45.66 & 4.71 & 5.45 & 4.91 & 8.72 & 20983 & 3795 & 10.4 & 5.2 \\
\hline $2016-07-17$ & 16.91 & 14.33 & 30.08 & 37.36 & 4.63 & 5.61 & 4.28 & 8.44 & 20971 & 3227 & 10.4 & 5.3 \\
\hline
\end{tabular}

TABLE 5: POD results for KOMPSAT-5 without GPS observations during attitude maneuvers.

\begin{tabular}{|c|c|c|c|c|c|c|c|c|c|c|c|c|}
\hline \multirow{3}{*}{ Date } & \multicolumn{12}{|c|}{ Case 2: edited measurement files } \\
\hline & \multicolumn{4}{|c|}{$\mathrm{RMS}_{\mathrm{RD}-\mathrm{KIN}}(\mathrm{cm})$} & \multicolumn{4}{|c|}{$\mathrm{RMS}_{\mathrm{RD}-\mathrm{SCI}}(\mathrm{cm})$} & \multirow{2}{*}{ \# input obs. } & \multirow{2}{*}{ \# marked obs. } & \multicolumn{2}{|c|}{$\begin{array}{c}\text { Residual. } \\
\text { (mm) }\end{array}$} \\
\hline & Radial & Along-track & Cross-track & $3 \mathrm{D}$ & Radial & Along-track & Cross-track & $3 \mathrm{D}$ & & & $\mathrm{RD}$ & KIN \\
\hline 2016-07-12 & 15.80 & 13.27 & 14.00 & 24.93 & 4.38 & 5.32 & 7.20 & 9.97 & 17755 & 967 & 10.1 & 5.4 \\
\hline 2016-07-13 & 15.56 & 13.29 & 13.90 & 24.74 & 4.98 & 5.80 & 4.55 & 8.90 & 17116 & 903 & 10.2 & 5.5 \\
\hline 2016-07-14 & 15.02 & 11.58 & 11.68 & 22.27 & 4.73 & 6.44 & 5.98 & 9.98 & 17703 & 957 & 10.0 & 5.4 \\
\hline 2016-07-15 & 15.01 & 12.49 & 11.35 & 22.59 & 4.55 & 6.08 & 7.11 & 10.40 & 17551 & 778 & 10.0 & 5.3 \\
\hline 2016-07-16 & 15.68 & 12.71 & 12.00 & 23.48 & 4.90 & 6.20 & 5.20 & 9.46 & 17638 & 871 & 10.1 & 5.4 \\
\hline $2016-07-17$ & 15.85 & 12.82 & 12.21 & 23.76 & 5.09 & 6.66 & 4.94 & 9.73 & 17533 & 793 & 10.0 & 5.4 \\
\hline
\end{tabular}

with those of previous studies pertaining to GRACE $[2,17$, 18]. The comparison results of RD-SCI indicate that the RD methods used in this study is well defined and the POD results are acceptable. Therefore, the quality of the signal itself can be investigated from the RD-KIN results. The measurement residuals of KIN are better than those of $\mathrm{RD}$ because the kinematic solutions are solely determined based on the GPS observations. The residual plots of GRACE-A in Figure 4 achieved from RD and KIN methods also show this feature. Figure 5 shows the position errors of the GRACE-A satellite in the radial, along-track, and crosstrack directions obtained on 14 July 2016 for RD-KIN and RD-SCI. It is evident that there are more fluctuations along with periodic variations for RD-KIN (Figure 5(a)) compared with RD-SCI (Figure 5(b)), which is likely because the kinematic positions reflect the observational properties directly without any smoothing effects like equations of orbital motions of the reduced-dynamic method. The periodic 


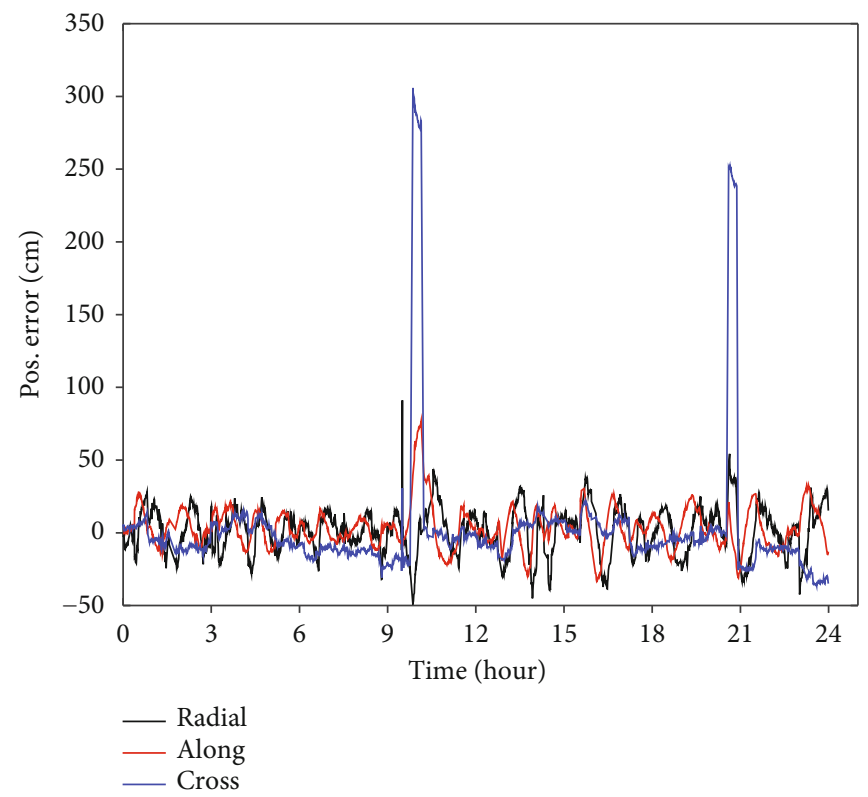

(a) RD-KIN (with raw observations)

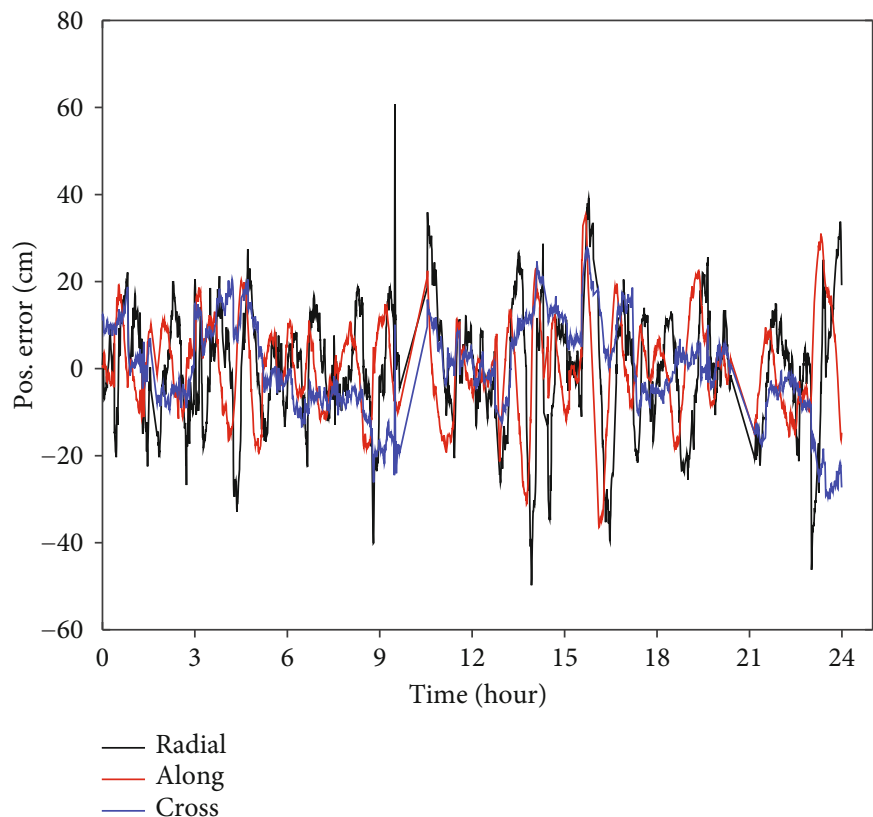

(b) RD-KIN (with edited observations)

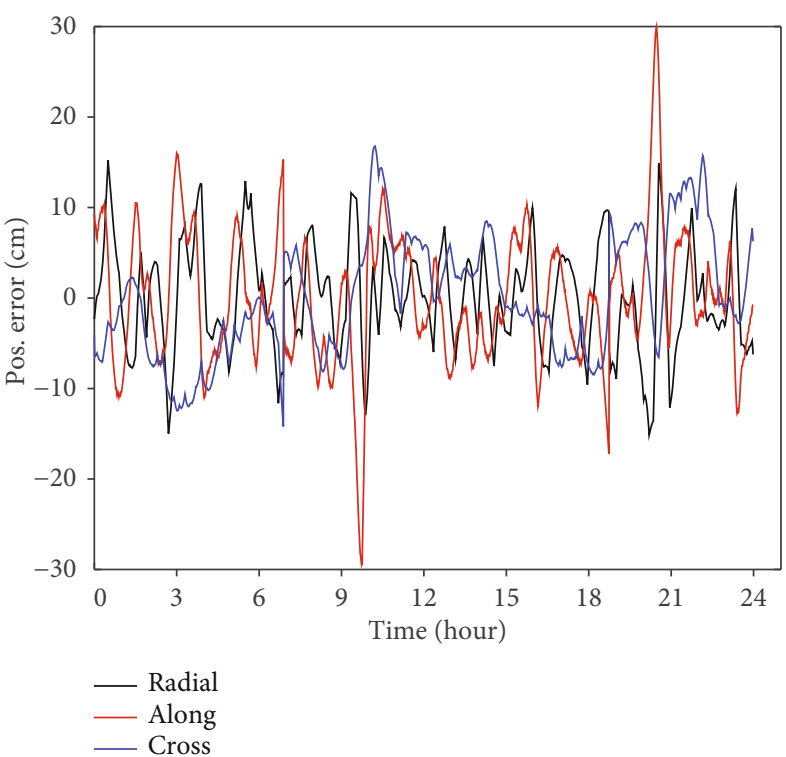

(c) RD-SCI

Figure 6: Position errors of the KOMPSAT-5 satellite in the radial, along-track, and cross-track directions on 14 July 2016.

variations in the radial and along-track directions in Figure 5(a) indicate that there is a need to gain a deeper understanding on the role of dynamic orbital models and measurement characteristics [2].

\section{Analysis of the POD Performances of KOMPSAT-5 and GRACE-A}

The orbital positions of the KOMPSAT-5 satellite were determined using the same POD scheme used for the GRACE-A satellite. The performance of the GPS-based POD system for KOMPSAT- 5 was investigated, and the results were ana- lyzed with those for GRACE-A, which will be presented and discussed in this section. The tests were performed for six days beginning from 12 July 2016 which are the same as the case of GRACE-A. First, the effect of frequent attitude maneuvers of KOMPSAT-5 on the POD performance was tested by comparing both the POD results obtained from the raw GPS observations including attitude maneuver periods and those obtained after excluding the GPS observations during attitude maneuvers. Second, the effect of the antenna type was investigated by comparing the results obtained for KOMPSAT-5 (without choke ring) with those for GRACE-A (with choke ring). 


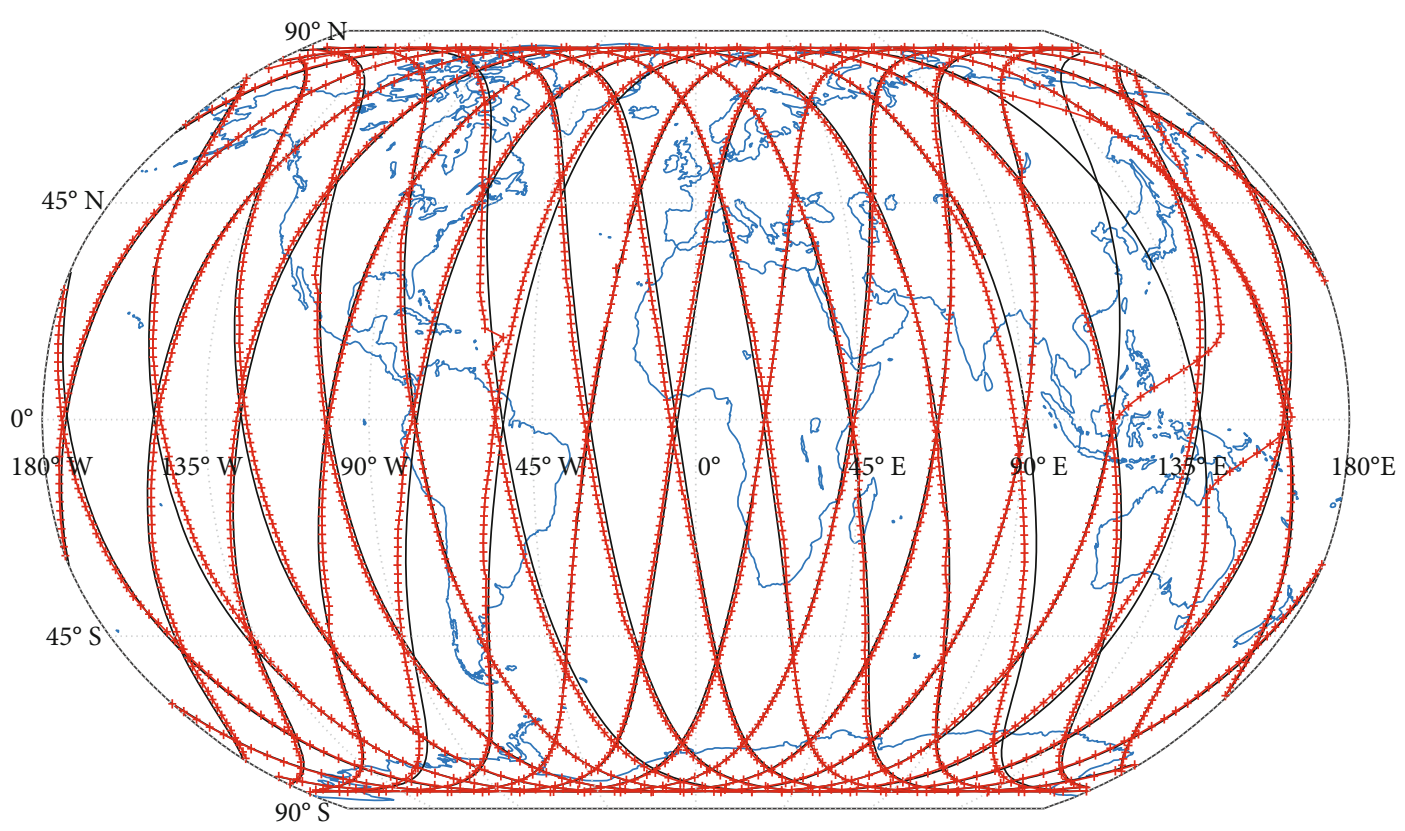

FiguRE 7: Ground track of KOMPSAT-5 (black) and the cross-track error of (RD-KIN) (red) for 14 July 2016.

4.1. Effects of Attitude Maneuvers. KOMPSAT-5 frequently performs attitude maneuvers for SAR imaging as the satellite passes above the Korean Peninsula. The start time and end time of the attitude maneuvers as well as the start time and end time of the measurement removal are summarized in Table 3. The average duration of the attitude maneuver is roughly 44 minutes, and the maneuver is typically performed two or three times depending on the number of times that the satellite passes above the Korean Peninsula. In order to examine the effect of attitude maneuvers on the POD results, the POD method was conducted using two types of observation files: (1) raw GPS observations and (2) edited GPS observations during the attitude maneuver. The GPS observations taken during attitude maneuvers were removed manually within a one-minute margin for before and after the actual maneuver time. The POD results for KOMPSAT-5 with and without removal of GPS observations during attitude maneuvers are tabulated in Tables 4 and 5, respectively. The numbers of raw and the rejected observations are also presented in Tables 4 and 5.

The average number of removed GPS observations is about 3,500 which constitutes $16 \%$ of the total number of observations. It can be seen from Table 5 that the removal of GPS observations during attitude maneuvers has a significant effect on the RMS errors of cross-track for RD-KIN whereas the effect is minor for RD-SCI. Therefore, it can be deduced that the kinematic method (which is solely based on GPS observations to determine the precise orbits of satellites) is susceptible to errors due to attitude maneuvers even though there is the preprocessing phase to screen GPS observations influenced by the attitude maneuvers. The SCI solutions represent the orbit products calculated by the University Corporation for Atmospheric Research (UCAR) with an accuracy of $10 \mathrm{~cm}$ [19]. The POD solution from UCAR is achieved as a part of radio occultation processing through Bernese S/W, and the detailed processing strategy can be referred in [19]. However, the POD using the reduceddynamic method absorbs the attitude maneuver errors by estimating the empirical acceleration along with the orbital elements; therefore, the reduced-dynamic method works properly regardless of removing the observations achieved during maneuvers. This conclusion can be also seen in Figure 6 that plots the RD-KIN orbit comparison results for Jul. 14, 2016 for the cases of with and without the observations during the attitude maneuvers in Figures 6(a) and 6(b), respectively. The RD-SCI position errors are also depicted in Figure 6(c) for a comparison purpose. The two peak errors in cross-track component are clearly shown in Figure 6(a), and these abnormal errors are not shown in the RD-SCI case in Figure 6(c). The location of attitude maneuvers and their effects on the GPS signals can be seen in Figure 7, which shows the cross-track error of RD-KIN obtained from the GPS observation including attitude maneuvers of Figure 6(a). The crosstrack position differences of centimeter level are intentionally scaled by a factor of 10 and changed a unit to degree to show the deviation as a longitudinal offset in Figure 7. Two large anomalies of the cross-track error of KOMPSAT-5 in Figure 6(a) are also shown in Figure 7 around the Korean Peninsula compared with those for other areas.

The most distinguishable difference between KOMPSAT5 and GRACE-A POD results is the number of observations. The number of raw GPS observations of KOMPSAT-5 is lower than that of GRACE-A by $4,000(\sim 16 \%)$. The actual number of observations used to determine the final orbit of satellite is from 16,000 to 17,000 which is $27 \%$ lower than that for GRACE-A (about 23,000 observations). Thus, the frequent attitude maneuvers of KOMPSAT- 5 have a significant effect on the POD performances. 


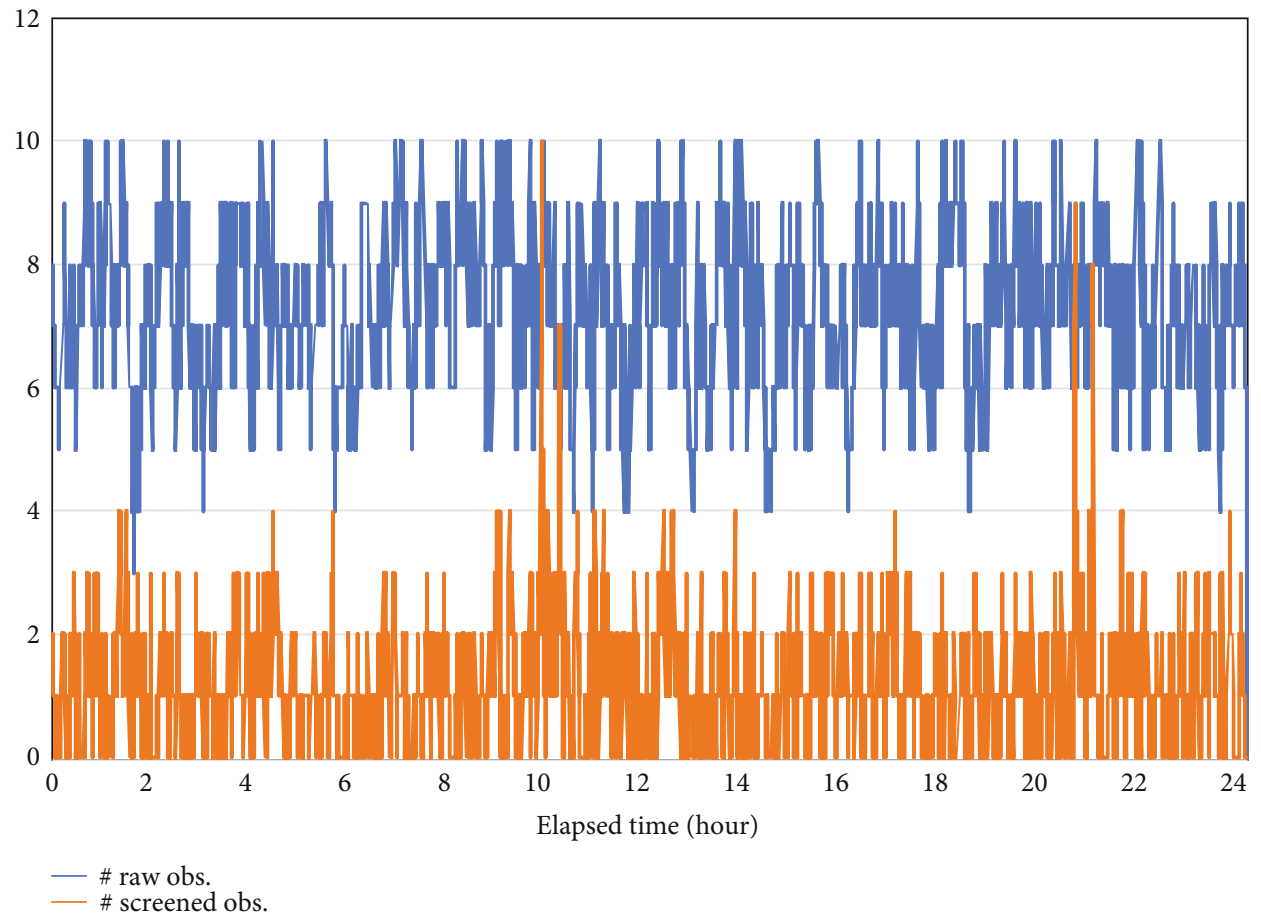

(a) KOMPSAT-5

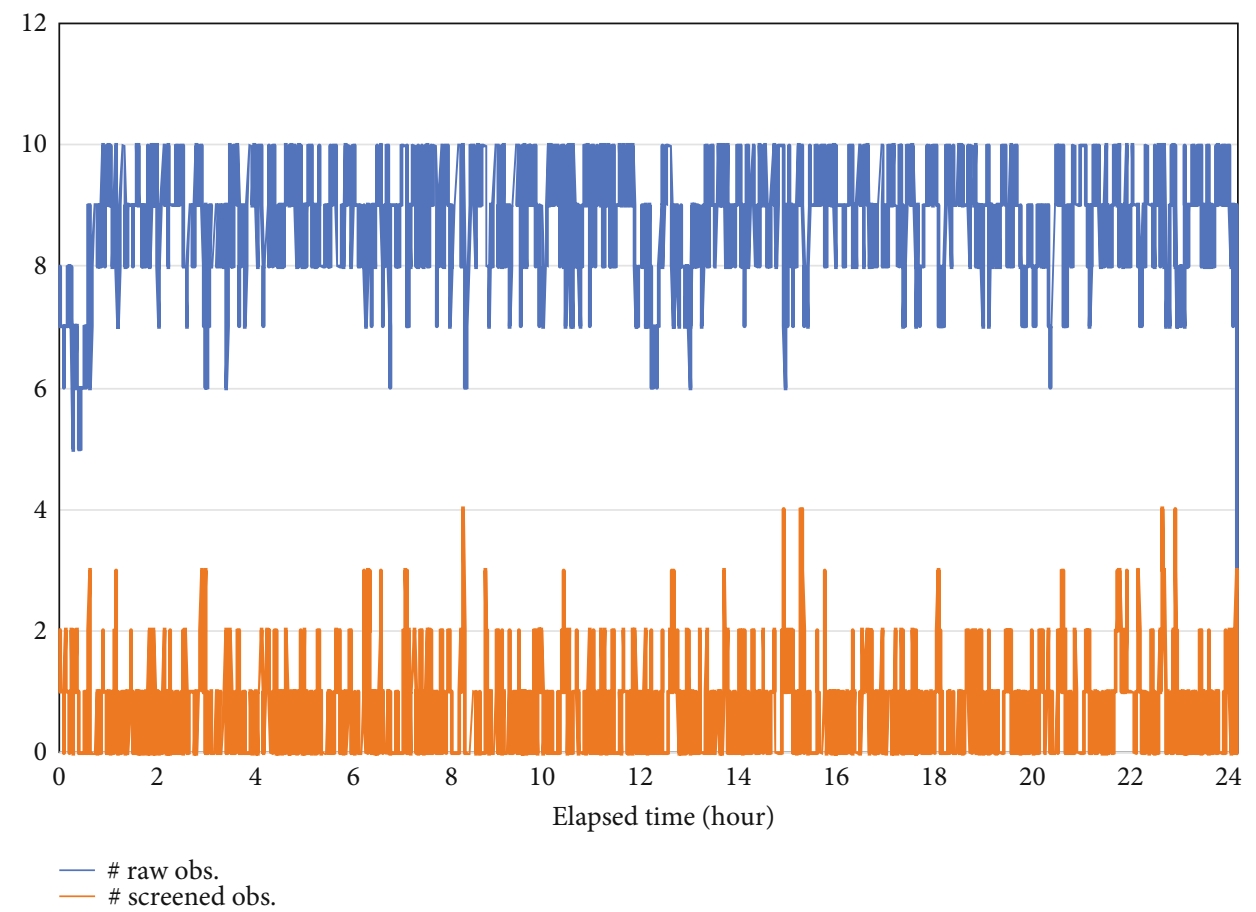

(b) GRACE-A

FIGURE 8: The numbers of raw and screened observations for KOMPSAT-5 and GRACE-A on 14 July 2016.

4.2. Effects of Antenna-Its Type and Geometrical Location. The total number of available observations of KOMPSAT-5 is significantly lower than that of GRACE-A as shown in the previous section even though the two satellites have similar GPS receivers. However, the overall quality of GPS signals is also poorer than the case of GRACE. In this subsection, the most possible reason of this poor quality is analyzed through investigating measurement residuals. Figure 8 shows the number of satellites tracked by the onboard patch GPS antennas of KOMPSAT-5 and GRACE-A along with the number of the screened number of observations mostly due to large measurement residual. The numbers displayed in Figure 8(a) are achieved from the raw observation data without removing the observations during the maneuvers. 


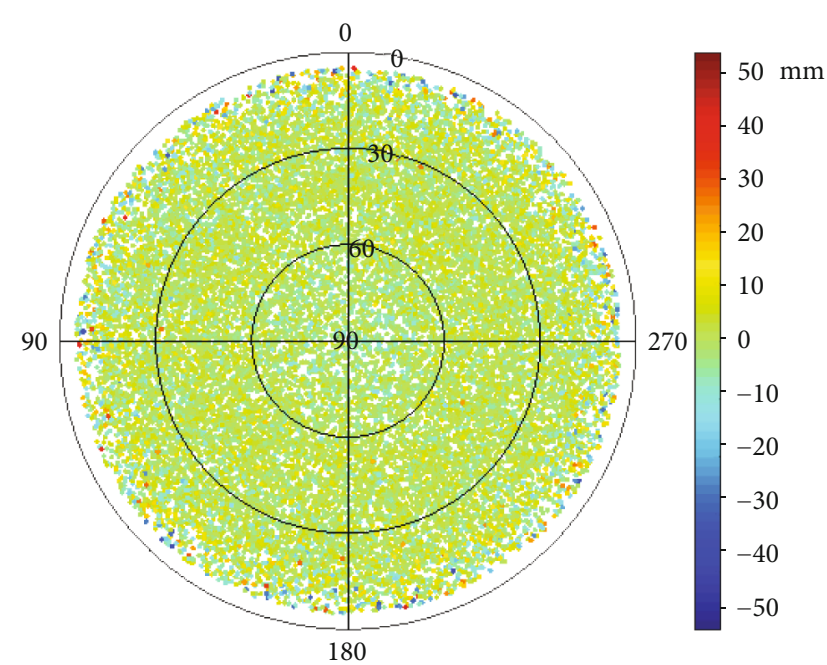

(a)

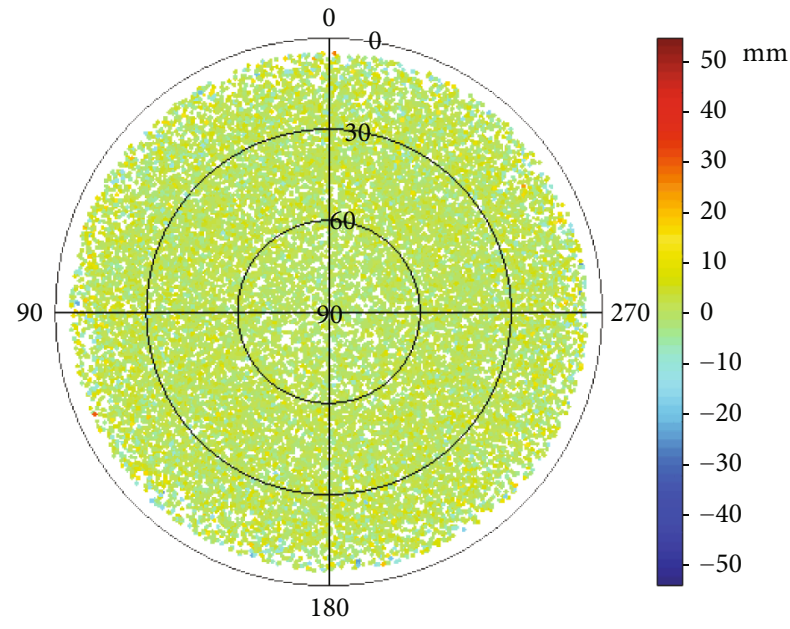

(b)

Figure 9: Measurement residuals for (a) KOMPSAT-5 and (b) GRACE-A on 14 July 2016.

There are two distinctive peaks in the number of screened observations due to errors caused by attitude maneuvers. Additionally, the number of raw observations and the number of screened observations of KOMPSAT- 5 have a larger variation than those in the case of GRACE-A. First, the discrepancy in the number of tracked satellites can be understood that the geometric position of the GPS antenna of KOMPSAT- 5 causes a geometrical blocking of GPS signals because the antenna on KOMPSAT- 5 is installed on the top side of the spacecraft along with other sensors (Figure 2) unlike in the GRACE case which is located at the top of the spacecraft without any interference with other instruments. Second, the differences in the number of screened observations are related to signal quality itself of both KOMPSAT5 and GRACE-A. As can be seen from the measurement residual plots in Figure 9, the measurement residuals of KOMPSAT- 5 are larger and show more fluctuations than those of GRACE-A. When taking into account that the two satellites have the same receiver, the most probable candidate for this discrepancy would be a type of antenna as well as its geometrical location. A patch GPS antenna of KOMPSAT-5 is advantageous because of its compact size. However, this type of antenna is susceptible to multipath errors compared with a choke ring antenna used on the GRACE-A satellite. The choke ring antenna is normally known to have strength to the multipath error. Besides, the measurement residuals of kinematic solutions and reduced-dynamic method are about $6 \mathrm{~mm}$ and $10 \mathrm{~mm}$ for GRACE-A (Table 2) and KOMPSAT- 5 (Table 4), respectively. In a low elevation angle, GPS observation of KOMPSAT-5 is rarer than those of GRACE because of the antenna location. This difference indicates that KOMPSAT- 5 has a poorer signal quality compared with GRACE-A as shown in Figure 9. It can be clearly observed that the measurement residuals of KOMPSAT- 5 are larger and more uneven than those of GRACE-A. Therefore, we deduce that the type of antenna used in KOMPSAT- 5 and its geometrical location are the major reasons for the erratic observations and low POD performance of KOMPSAT- 5 .

\section{Conclusions}

The KOMPSAT- 5 satellite is equipped with a GPS receiver for POD and radio occultation study, which is the secondary task of the satellite. The objective of this study is to predict the POD performance of satellites with GPS-based POD systems, where POD is not the primary mission of the satellite such as KOMPSAT-5. The primary mission of KOMPSAT-5 is to acquire SAR images, and frequent attitude maneuvers take place during this mission. A patch GPS antenna is installed onboard of KOMPSAT- 5 to balance with other payloads, which differs from the choke ring GPS antenna used in the GRACE satellites. The effects of the antenna type and operational strategies on the POD accuracy were analyzed. The POD scheme developed in this study was applied to the observations of the GRACE-A satellite, which is a typical satellite used for geodetic missions. The results showed that the achievable orbital position accuracy is $2-3 \mathrm{~cm}$ compared with the GRACE Level-1B product. The reduceddynamic method was used to determine the orbital positions of KOMPSAT-5, and the solutions were compared with the products released by UCAR. The RMS error of the orbital position difference is $\sim 10 \mathrm{~cm}$, and the values are not affected by including the GPS observation data during attitude maneuvers. However, the orbital position discrepancies between the reduced-dynamic and kinematic methods improved from $50 \mathrm{~cm}$ to $25 \mathrm{~cm}$ by excluding observations during attitude maneuvers. Therefore, it can be concluded that the GPS signals of KOMPSAT- 5 are influenced by attitude maneuvers. The position and type of GPS antenna also affect the GPS signals of KOMPSAT-5, where the number of observations per day of KOMPSAT- 5 is $\sim 80 \%$ of that for GRACE-A and the actual number of observations used for POD after screening is $\sim 70 \%$ of that for GRACE-A. This discrepancy is primarily due to the fact that the operational strategy and design of the KOMPSAT-5 satellite are not optimized for POD with a high accuracy. The results of this study showed that the actual performance of the GPS-based POD 
system is dependent on the operational strategy and hardware design of the mission.

\section{Data Availability}

The KOMPSAT-5 and GRACE data used to support the findings of this study have been deposited in the KASI repository (ftp://aopod-ftpkasi.re.kr) and NASA (https://podaac.jpl .nasa.gov/dataset/GRACE_L1B_GRAV_JPL_RL03).

\section{Conflicts of Interest}

The authors declares that they have no conflicts of interest.

\section{References}

[1] H. Bock, A. Jäggi, D. Švehla, G. Beutler, U. Hugentobler, and P. Visser, "Precise orbit determination for the GOCE satellite using GPS," Advances in Space Research, vol. 39, no. 10, pp. 1638-1647, 2007.

[2] A. Jäggi, U. Hugentobler, H. Bock, and G. Beutler, "Precise orbit determination for GRACE using undifferenced or doubly differenced GPS data," Advances in Space Research, vol. 39, no. 10, pp. 1612-1619, 2007.

[3] S.-R. Lee, "Overview of KOMPSAT-5 program, mission, and system," in IGARSS 2010-2010 IEEE International Geoscience and Remote Sensing Symposium, pp. 797-800, Honolulu, HI, USA, July 2010.

[4] Y. Hwang, B. Lee, Y. Kim, K. Roh, O. Jung, and H. Kim, "GPSbased orbit determination for KOMPSAT-5 satellite," ETRI Journal, vol. 33, no. 4, pp. 487-496, 2011.

[5] A. Jäggi, R. Dach, O. Montenbruck, U. Hugentobler, H. Bock, and G. Beutler, "Phase center modeling for LEO GPS receiver antennas and its impact on precise orbit determination," Journal of Geodesy, vol. 83, no. 12, pp. 1145-1162, 2009.

[6] O. Jung, A. Chung, E. Kim, J. Yoon, and Y. Hwang, "Analysis on the orbit accuracy of KOMPSAT-5," Aerospace Engineering and Technology, vol. 13, no. 2, pp. 108-114, 2014.

[7] GFZ-Potsdam, "GFZ-Potsdam," 2018.

[8] R. Kroes, O. Montenbruck, W. Bertiger, and P. Visser, "Precise GRACE baseline determination using GPS," GPS Solutions, vol. 9, no. 1, pp. 21-31, 2005.

[9] Z. Kang, B. Tapley, S. Bettadpur, J. Ries, P. Nagel, and R. Pastor, "Precise orbit determination for the GRACE mission using only GPS data," Journal of Geodesy, vol. 80, no. 6, pp. 322-331, 2006.

[10] eoPortal, Earth observation portal: KOMPSAT-5, eoPortal, 2018.

[11] R. Dach, S. Lutz, P. Walser, and P. Fridez, Bernese GNSS software version 5.2., R. Dach, S. Lutz, P. Walser, and P. Fridez, Eds., University of Bern, Bern Open Publishing, Bern, 2015.

[12] A. Jäggi, U. Hugentobler, and G. Beutler, "Pseudo-stochastic orbit modeling techniques for low-earth orbiters," Journal of Geodesy, vol. 80, no. 1, pp. 47-60, 2006.

[13] B. Zhang, Z. Wang, L. Zhou, J. Feng, Y. Qiu, and F. Li, "Precise orbit solution for swarm using space-borne GPS data and optimized pseudo-stochastic pulses," Sensors, vol. 17, no. 3, p. 635, 2017.

[14] K. Case, G. Kruizinga, and S.-C. Wu, GRACE Level $1 B$ data product user handbook, JPL Publication D-22027, 2010.
[15] R. Dach, S. Schaer, D. Arnold, L. Prange, D. Sidorov, and A. Susnik, CODE Final Products Series for the IGS, Astronomical Institute, University of Bern, 2018.

[16] K.-M. Roh and B.-K. Choi, "The effects of the IERS conventions (2010) on high precision orbit propagation," Journal of Astronomy and Space Sciences, vol. 31, no. 1, pp. 41-50, 2014.

[17] Z. Kang, B. Tapley, S. Bettadpur, J. Ries, and P. Nagel, "Precise orbit determination for GRACE using accelerometer data," Advances in Space Research, vol. 38, no. 9, pp. 2131-2136, 2006.

[18] N. Zehentner and T. Mayer-Gürr, "Precise orbit determination based on raw GPS measurements," Journal of Geodesy, vol. 90, no. 3, pp. 275-286, 2016.

[19] Y. Yoon, J. Weiss, D. Hunt, T. Vanhove, and M. Sleziak-Sallee, "Upgrading the CDAAC post- and re-processing LEO orbit products and its impact on GPS RO retrieval," in International Conference on GPS RO, pp. 1-19, Taipei, Taiwan, 2018. 